\title{
The Use of High Speed, High Resolution EBSD to Unlock Hidden Secrets of the Allende Meteorite
}

\author{
Pat Trimby ${ }^{1}$, Luke Daly ${ }^{2}$ and Sandra Piazolo ${ }^{3}$ \\ 1. Oxford Instruments Nanoanalysis, High Wycombe, U.K. \\ 2. School of Geographical and Earth Sciences, University of Glasgow, UK \\ 3. School of Earth and Environment, University of Leeds, UK
}

Electron backscatter diffraction (EBSD) in conjunction with integrated energy dispersive X-ray spectrometry (EDS) has been routinely used to characterize geological and extraterrestrial samples for more than two decades. However, the complex microstructures that exist in many meteorite samples, and especially carbonaceous chondrites, pose a particular challenge for successful microstructural characterization. Typically structures exist that require resolution on a sub- $\mu \mathrm{m}$ scale yet display heterogeneity on a mm scale. Although there have been some detailed conventional EBSD analyses of carbonaceous chondrite samples [e.g. 1], these have either focused on small targeted regions or have sacrificed spatial resolution to ensure coverage of a representative area, and have in all cases taken significantly more than 100 hours of analysis time. More detailed analyses of small scale features (such as $\mu \mathrm{m}$-scale refractory metal nuggets) have been carried out using a recently developed variant of EBSD, transmission Kikuchi diffraction (TKD) [2], yet once again such studies are time and instrument intensive and may not be fully representative of sample-scale variations.

Recent developments in EBSD detector technology have resulted in a dramatic increase in data acquisition speed, especially for more complex materials such as minerals and ceramics. The use of a complementary metal oxide semiconductor (CMOS) sensor instead of a more conventional charge coupled device (CCD) sensor enables the collection of good resolution diffraction patterns at speeds in excess of 3000 patterns per second (pps) for metals and 1000 pps for minerals [3]. In addition, the use of large area EDS detectors (e.g. $>150 \mathrm{~mm}^{2}$ ) provides excellent X-ray count rates and allows the simultaneous collection of high quality chemical data even at these high collection rates. The implications of these technological advances are profound: it is now possible to analyze complex, heterogeneous carbonaceous chondrite microstructures with sufficient resolution to interpret the finest scale features, yet covering $\mathrm{mm}^{2}$ areas to capture localised heterogeneities, all within a few hours.

In this paper we present results from the Allende CV 3.2 meteorite that show the potential of this high speed, high resolution approach to aid understanding of the processes involved in the meteorite's deformation and metamorphic history. A polished block was analysed in a Hitachi SU-70 field emission scanning electron microscope (FE SEM) using an Oxford Instruments AZtec integrated EBSD-EDS system equipped with an X-Max $150 \mathrm{~mm}^{2}$ EDS detector and a Symmetry CMOS-based EBSD detector. EBSD patterns were collected with a resolution of 156x128 pixels at speeds between 500 and 1208 pps, with live indexing to provide both orientation and phase data. Analyses were carried out using measurement spacings between $2 \mu \mathrm{m}$ and $120 \mathrm{~nm}$, providing sufficient resolution to examine in detail the fine structures in both the Allende olivine-rich matrix, the chondrules and calcium aluminium rich inclusions (CAIs).

Typical results are shown in figure 1. Large scale, high resolution EBSD maps consisting of 10s millions measurements resolve structures within coarser-grained regions such as chondrules as well as the fine 
grained, porous matrix (figure 1a). Detailed examination of the crystallographic features and chemical variations reveal a number of interesting phenomena. For example, some areas within the fine grained olivine matrix show a significant number of twinned grains $\left(60^{\circ}\right.$ rotation about $<100>$; figure $\left.1 \mathrm{~b}\right)$. The implication of these twins in olivine is unclear, but they may be related to a topotactic regressive phase transformation from the high-pressure cubic polymorph, ringwoodite [4]. The occurrence of ringwoodite in shocked chondritic meteorites has been extensively reported, usually associated with shocked vein structures, providing shock pressure estimates of up to $20 \mathrm{GPa}$ [5]. There has been no previously reported evidence for ringwoodite in the Allende meteorite. We also observe significant chemical zonation in olivine grains at the margins of chondrules and CAIs (e.g. figure 1c), as well as evidence for replacement of clinopyroxene by olivine and associated diffusion of $\mathrm{Ca}$ across distances of 5-10 $\mu \mathrm{m}$. Thermodynamic calculations allow us to assign a timescale for this diffusion on the order of 1 day, providing additional constraints for the deformation events associated with meteorite compaction.

The results here show the potential of large scale, high speed characterization of meteorite samples using EBSD and EDS. We will discuss how these data from the Allende meteorite have enabled us to advance our understanding of compaction and shock events of this extensively studied meteorite, and will look at the implications for future microstructural analyses of samples in the planetary sciences.

\section{References:}

[1] L. Forman et al., Geology 45 (2017) p. 559-562.

[2] L. Daly et al., Geochim. Cosm. Acta 216 (2017), p. 42-60.

[3] www.oxinst.com/symmetry

[4] L. Kerschhofer et al., Min. Mag.62 (1998), p. 617-638.

[5] M. Chen, A. Goresy and P. Gillet, PNAS 101 (2004), p. 15033-15037.

[6] The authors acknowledge the input from Phil Bland, Lucy Forman and Geoff Lloyd.


Figure 1. Example EBSD-EDS maps of the Allende meteorite (a) Inverse pole figure ( $\mathrm{Y}$ direction) orientation map showing fine grained olivine matrix grains surrounding coarser-grained inclusions. Map collected with $200 \mathrm{~nm}$ spacing at 520 pps. Scale bar: $200 \mu \mathrm{m}$. (b) Phase map of area marked in (a): red olivine, blue - clinopyroxene, purple - FeNi sulfide. Yellow lines mark twin boundaries within olivine grains (area marked by white arrow). Scale bar: $40 \mu \mathrm{m}$. (c) Mg element map of same area showing zonation within olivine grains in the coarse inclusion (e.g. as marked by white arrow). Scale bar: $40 \mu \mathrm{m}$. 